\title{
Analysis of the localization process leading to fragmentation of dy- namically expanded rings
}

\author{
Skander El Mai ${ }^{1, *}$, Sébastien Mercier ${ }^{2}$, and Alain Molinari ${ }^{2}$ \\ ${ }^{1}$ CEA, DAM, Gramat, F-46500, France \\ ${ }^{2}$ LEM3, Université de Lorraine - UMR CNRS 7239, BP 15082, 57073 Metz Cedex 03, France
}

\begin{abstract}
A novel extension of the Linear Stability Analysis (LSA) applied to the development of the instabilities in a round bar in dynamic extension is proposed. The history of development of the instabilities and the multi-modal aspect of the perturbations are now considered in this extension. A new statistical approach based on the power spectrum of the perturbation pattern is proposed to evaluate the variability of the spacing between potential sites of necking. This approach has been applied to analyze the LSA results and predictions obtained from numerical simulations of a bar in dynamic extension. It is shown that both analytical and numerical approaches provides good agreement.
\end{abstract}

\section{Introduction}

The understanding of fragmentation process of metallic casing (usually made of steel) is an important issue for civil, industrial and military purposes. Fragmentation can be observed during hazardous event like explosion of pipes due to temporary overloading, of pressurized cylinders containing fluid or industrial gas or ignition of ammunition for defense applications. The explosion of the structure will generate in some situations, parts of various sizes flying at large velocity. The control of the fragment distribution is critical. From the literature survey, this phenomenon is still of interest following two main modeling pathways: an approach based on Mott [1], Taylor [2] and Grady's [3] theories and a purely numerical approach which utilizes perturbations. These studies have shown a strong potential for predicting the fragment distribution in both cases. Fragmentation of ductile casing is a two stage process with first the occurrence of localized strain zones followed by fracture.

The present work focuses on the development of localization based on a novel multi-modes analysis applied to the evolution of the cross-section of a bar in dynamic tension with an initial surface roughness. Section 2 describes the principles of this new modeling $[4,5]$ based on the linear stability analysis developed in [6]. In Section 3, new outcomes obtained from the present approach are analysed.

*e-mail: skander.elmai@cea.fr

\section{Linear Stability Analysis for the prediction of a mean neck spacing}

Mercier and Molinari developed in [6] a 3D linear stability analysis (LSA) for a bar in tension (Fig. 1), accounting for inertial pressure together with stress triaxiality effects for rate-insensitive and rate-sensitive materials with strain hardening. This analysis is dedicated to the characterization of the instantaneous growth rate $\eta$ of modal perturbations (proportional to $\sin \left(k_{p} Z\right)$, where $k_{p}$ is the wavenumber of the perturbation). The analysis has some limitations since [6] considers that the most unstable mode will define the necking pattern while experimental observations show that necking is a statistical process with different inter-neck distances. In addition, the time evolution of the perturbations is not really taken into account since the perturbation is not tracked during the whole deformation process.

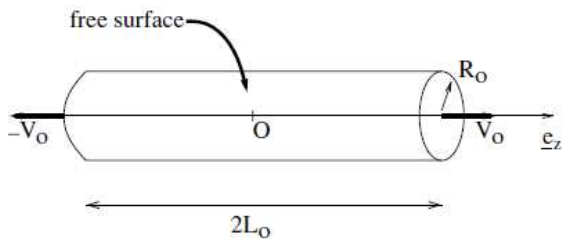

Fig. 1. Schematic representation of the cylindrical bar, of initial length $2 L_{0}$ and of initial radius $R_{0}$. Velocities $\pm V_{0}$ are applied to the extremities. The lateral surface is traction free. 


\subsection{Configuration of interest}

The configuration of $[4,5]$ is considered (see Fig. 1): a cylindrical copper bar, of initial length $2 L_{0}=128.805 \mathrm{~mm}$ and circular cross-section $S_{0}=1 \mathrm{~mm}^{2}$ is subjected to uniform and constant velocity $V_{z}= \pm V_{0}$, applied at the extremities $\left(Z= \pm L_{0}\right)$. The material response of copper integrates hardening, strain rate and temperature effect and a modified Zerilli-Amstrong flow law is adopted, as in [4]. The reference configuration is based on a constant loading velocity $V_{0}=900 \mathrm{~m} / \mathrm{s}$, so the nominal strain rate is around $14 \times 10^{3} \mathrm{~s}^{-1}$. The influence of $V_{0}$ on the development of instabilities is also analysed for two additional loading velocities: $150 \mathrm{~m} / \mathrm{s}$ and $3000 \mathrm{~m} / \mathrm{s}$.

\subsection{Cross-section perturbation evolution}

In the present work, the reference configuration is perfectly cylindrical with a uniform radius $R_{0}$. This study is interested in the description of time evolution of a perturbation pattern during dynamic tension. For that purpose, an initial cross-section perturbation is considered and described by a Fourier series expression given by:

$$
\delta S_{0}(Z)=\sum_{p \in \mathbb{N}-\{0\}} \delta S_{0}^{p} \cos \left(k_{p} Z+\varphi_{p}\right)
$$

where $\delta S_{0}^{p}$ is the amplitude (Fourier coefficient) of mode $p$ associated to the wave-number $k_{p}=$ $p \frac{\pi}{L_{0}}$. The phase $\varphi_{p}$ is supposed to be an independent random number in the range $[0,2 \pi[$. Time evolution of each cross-section perturbation mode is evaluated considering that each perturbation mode grows independently. Based on these assumptions, time evolution of the cross-section perturbation of mode $p$ has been expressed in $[4,5]$ by:

$\delta S^{p}(Z, t)=\delta S_{0}^{p} \exp \left[\int_{0}^{t} \eta\left(k_{p}, \tilde{t}\right) d \tilde{t}\right] \cos \left(k_{p} Z+\varphi_{p}\right)$

which leads to the time evolution of the overall cross-section perturbation in terms of the Fourier series expression:

$\delta S(Z, t)=\sum_{p \in \mathbb{N}-\{0\}} \delta S_{0}^{p} \exp \left[\int_{0}^{t} \eta\left(k_{p}, \widetilde{t}\right) d \vec{t}\right] \cos \left(k_{p} Z+\varphi_{p}\right)$

where the time integral of the growth rate $\exp \left[\int_{0}^{t} \eta\left(k_{p}, \widetilde{t}\right) d \vec{t}\right]$ reproduces the amplitude evolution of mode $p$.

\subsection{Potential necking sites distribution}

Two approaches have been proposed in $[4,5]$ to analyze the potential neck (otherwise called prenecks) spacing distribution, during cross-section perturbation evolution:

1. the amplitude threshold method: this method is based on counting the number of valleys deeper than a pre-defined threshold, observed on a cross-section perturbation profile. The corresponding distance between neighbour valleys is a direct outcome of the strategy;

2. the power spectrum based method (PSD): this approach has been proposed and developed in details in $[4,5]$. It provides a probability density of pre-neck spacing based on the Power Spectral Density (PSD) representation of the cross-section perturbation. Comparisons with the previous method, conducted in [4], have validated this approach.

The PSD methodology has been employed to analyse the evolution of the cross-section perturbation by considering first an initial white noise perturbation, where the amplitude of all modes is identical, i.e. $\delta S_{0}^{p}=\delta S_{0}, \forall p$. As illustrated in Fig. 2(a) for a loading velocity of $V_{0}=900 \mathrm{~m} / \mathrm{s}$, with this particular initial perturbation, the probability density evolves gradually with three well defined stages :

1. an exponential shape at early times related to the flat spectrum of the white noise,

2. a bi-exponential shape at intermediate times,

3. a Weibull shape at late times.

In this study, the pre-neck spacing distributions are plotted with respect to the normalized distance $\hat{l}=l / L_{0}$. Each $\hat{l}$ may be associated to a fictitious number of pre-necks $n=2 / \hat{l}$. From this relation, the long term number of necks can be evaluated from the dominant normalized distance $\hat{l}_{\text {dom }}$ associated to the distribution maximum. Here, $n_{\text {dom }}=2 / \hat{l}_{\text {dom }} \approx 80$ at $t=80 \mu \mathrm{s}$. Same type of probability density evolution is retrieved for other loading velocities. Besides, it is observed that the long term Weibull-like distribution obtained from this approach is shifted in direction of lower neck spacing and its width is reduced, as illustrated in Fig.2(b) where are superimposed long term distributions for the three loading velocities $V_{0}=$ 150,900 and $3000 \mathrm{~m} / \mathrm{s}$ at respectively $t=300,80$ and $30 \mu s$. 


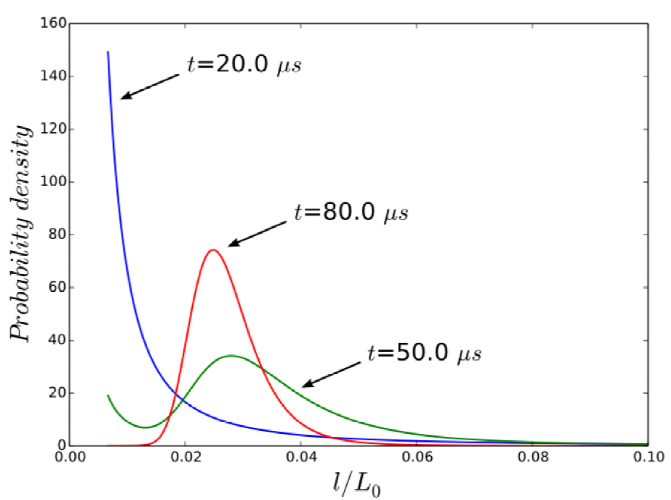

(a) Pre-neck spacing probability density at $V_{0}=900 \mathrm{~m} / \mathrm{s}$.

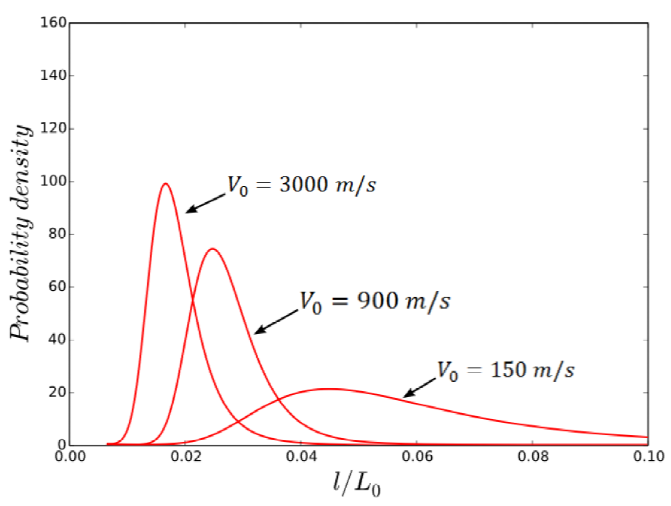

(b) Long term pre-neck spacing distributions at $V_{0}=150,900$ and $3000 \mathrm{~m} / \mathrm{s}$ at respectively $t=300,80$ and $30 \mu \mathrm{s}$.

Fig. 2. Evolution of (a) pre-neck spacing probability density at $V_{0}=900 \mathrm{~m} / \mathrm{s}$, and (b) comparison of long term pre-neck spacing distributions at loading velocities $V_{0}=150,900$ and $3000 \mathrm{~m} / \mathrm{s}$ at respectively $t=300,80$ and $30 \mu \mathrm{s}$. An initial white noise perturbation is considered for the dynamic extension of the bar.

The trends depicted in Fig. 2 for the pre-neck spacing distributions obtained from the power spectrum based method with an initial white noise perturbation are in agreement with experimental observations. One may refer to [7] where distributions of distances between necks for different ring expansion velocities are presented.

\section{Outcomes of the model}

In the following, we propose to perform some comparisons with results obtained from direct finite element (FE) simulations. In addition, the analysis of the numerical results obtained by [8] from pre-neck distributions, based on the proposed approach and considering specific initial cross-section perturbations [5], is suggested.

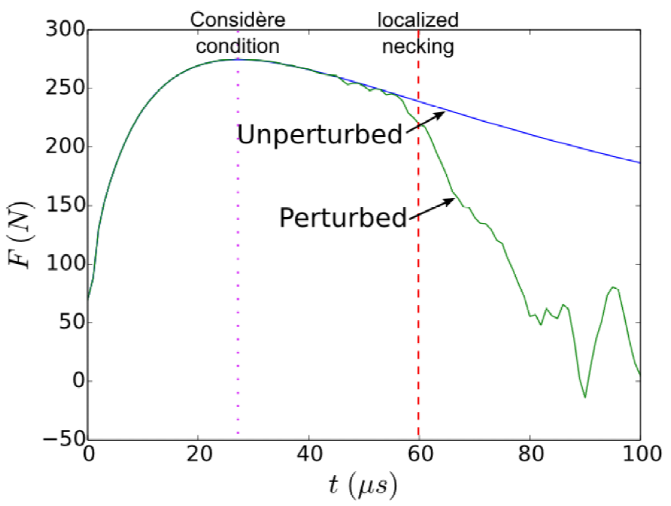

Fig. 3. Evolution of the traction force during the extension of the bar. The loading velocity is $V_{0}=900 \mathrm{~m} / \mathrm{s}$. The initial surface roughness is defined from Eq. 4 with $\delta R_{0}=54 \mu \mathrm{m}$.

\subsection{Comparisons with numerical results}

\subsubsection{Numerical configuration}

The power spectrum based method is applied here to process numerical simulations based on the configuration of section 2.1. Calculations are performed with the OURANOS code, developed by CEA. The three loading velocities $V_{0}=150,900$ and $3000 \mathrm{~m} / \mathrm{s}$ are considered. In order to trigger plastic instability (multiple necking) during the extension, it is assumed, in the present numerical simulations, that the bar has an initial surface roughness described by the following perturbation of the initial cross-section radius:

$$
\delta R=\delta R_{0} \sum_{p=1}^{N=600} \cos \left(k_{p} Z+\varphi_{p}\right)
$$

where $\delta R_{0}$ is an initial amplitude, $\varphi_{p}$ a random number in the range $\left[0,2 \pi\right.$ [ and $k_{p}=p \frac{\pi}{L_{0}}$ the wavenumber of mode $p$. In this way, the surface roughness of the bar is considered as a white noise. For the present paper, the initial amplitude $\delta R_{0}$ is set to $54 \mu \mathrm{m}$. In order to illustrate the influence of the perturbations on the bar in dynamic extension, one can follow the evolution of the traction force measured at the extremities $Z= \pm L_{0}$ during the loading. A comparison between a perfect round bar and a bar with an initial roughness (4) is given in Fig. 3 for the loading velocity $V_{0}=900 \mathrm{~m} / \mathrm{s}$. Similar responses have been obtained with the other two loading velocities $(150$ and $3000 \mathrm{~m} / \mathrm{s})$. A bifurcation in the force evolution is observed for the perturbed bar at around $60 \mu \mathrm{s}$. Indeed the perturbations develop with time and trigger a multiple necking pattern. Before bifurcation, the force evolution is identical for both perturbed and unperturbed configurations. 


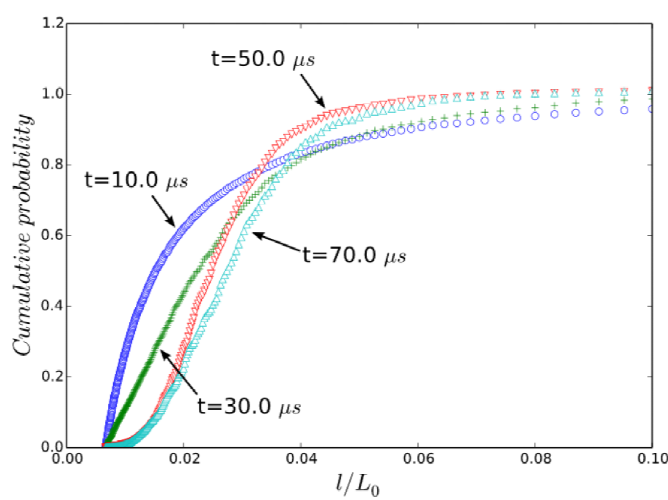

(a) Results from Numerical simulations

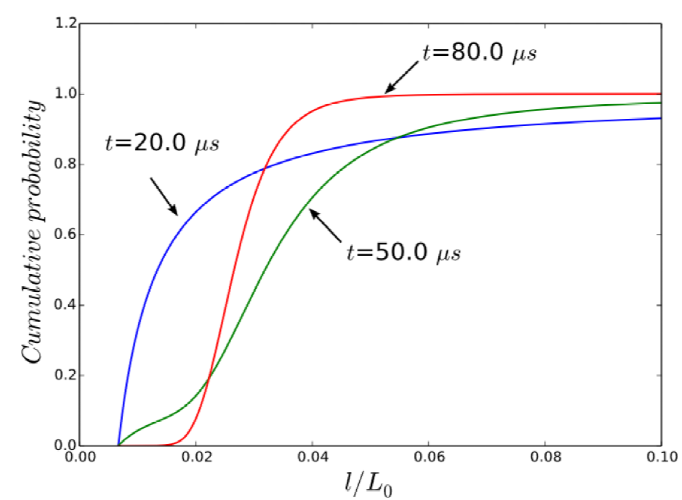

(b) Results predicted by the present linear stability analysis

Fig. 4. Pre-neck spacing cumulative probability at different stages of the deformation process. Predictions of a) direct finite element simulation (b) the proposed extension of the linear stability analysis. An initial white noise is adopted for the cross-section perturbation. The loading velocity is set to $V_{0}=900 \mathrm{~m} / \mathrm{s}$.

To analyze the evolution of the perturbations in the numerical simulations, the power spectrum of the cross-section perturbation profile is carried out at different times by slicing up the bar at 3000 equally distributed positions. The discrete Fast Fourier Transform (FFT) algorithm is used to calculate the power spectrum. The cumulative probability is presented in Fig. 4. Predictions based on the present model and on the numerical simulations are compared. It is observed that the profiles deduced from both strategies are really similar. The mean pre-neck spacing can be evaluated and it is also pointed out that both approaches provide similar values $\left(l_{\text {mean }} \approx 0.02 \times L_{0}\right)$. Nevertheless, some discrepancies exist on their time evolution. This is amplified when considering low velocity loading. Before the maximum of the force (as shown in Fig. 3), no positive growth rate for any perturbation mode is expected for the LSA. So the perturbation profile is frozen, while during numerical simulations, the perturbation amplitude is changing.

\subsection{Effects of a dominant initial perturbation mode}

A comprehensive numerical analysis of necking in a ring in dynamic expansion influenced by calibrated initial perturbations is well documented in [8]. One of the major results of [8], schematically illustrated in Fig.5, is the non trivial link between the final number of necks $n$ and the initial number of defects $N$. Five distinguishable regions can be determined:

- Regions I and III are characterized by the fact that $n$ is nearly constant and is driven by a natural dominant necking mode.
- Region II is represented by a straight line corresponding to the equation $n=N$ which suggests that the number of imperfections $N$ scales the final number of necks.

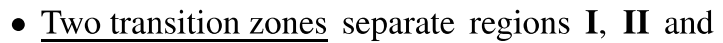
III.

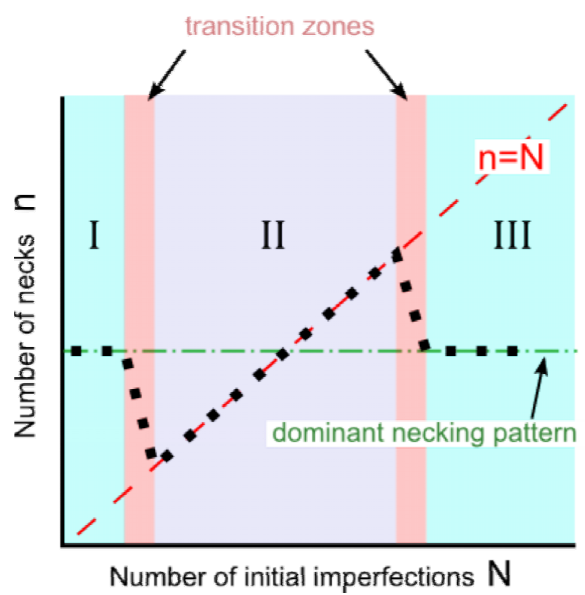

Fig. 5. Schematic representation of the necking behaviour observed by [8] stemmed by numerical results (black dotted curve). After the insertion of a number $N$ of geometrical imperfections in a ring in dynamic expansion, a number $n$ of necks is counted. Depending on $N$, for a given strain rate and amplitude of imperfections, two regions (I and III) present a fixed number of necks $n$ associated to a dominant necking pattern. Region (II) displays a different trend, where the number of necks is directly related to the initial flaws by the simple relationship : $n=N$, i.e. all the inception points develop into a neck.

In order to perform a comprehensive analysis of these results, initial perturbations $\delta S_{0}^{p}$ of Eq. (3) 


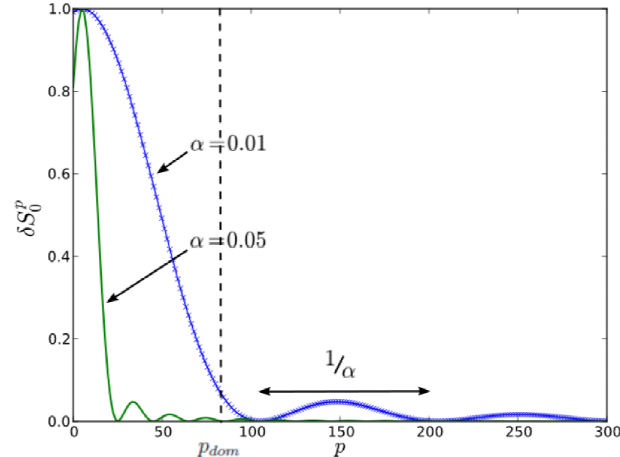

Fig. 6. Perturbation amplitude $\delta S_{0}^{p}$ described by the square sine cardinal function (5). The following parameters are adopted : $\delta S_{0}=1, N_{0}=5$ and two values of $\alpha$ : $\alpha=0.01$ and 0.05 .

have been constructed based on square sine cardinal spectrum :

$$
\begin{aligned}
\delta S_{0}^{p} & =\delta S_{0} \operatorname{sinc}^{2}\left(\alpha \pi\left(p-N_{0}\right)\right) \\
& =\delta S_{0}\left(\frac{\sin \left(\alpha \pi\left(p-N_{0}\right)\right)}{\alpha \pi\left(p-N_{0}\right)}\right)^{2}
\end{aligned}
$$

The square sine cardinal function enables to distribute the initial dominant perturbation around modes close to the mode $N_{0}$. Therefore, some modes, close to $N_{0}$ will have a significant initial amplitude. The range of initial dominant modes is highly related to the parameter $\alpha$, as seen in Fig. 6. Nevertheless, even for mode $p$ far from $N_{0}$, the amplitude is non zero so theses modes may evolve during loading and may become pre-dominant after some time.

Table 1 summarizes the dominant pre-neck spacing evaluated at different stages of the loading. Five configurations (one white noise and four perturbations based on the square sine cardinal) are compared. The reference white noise configuration leads to a dominant pre-neck distance $l_{\text {dom }}$, generating a number of necks $n_{\text {dom }}=2 L_{0} / l_{\text {dom }}$. With the specific pertubations based on Eq.(5), it is observed that when $N_{0}$ is chosen far from $n_{d o m}$ (here $n_{d o m}=80$ ), the number of necks will be strongly different from $N_{0}$. For the three cases $\left(N_{0}=5\right.$, $\alpha=0.05),\left(N_{0}=5, \alpha=0.01\right)$ and $\left(N_{0}=250\right.$, $\alpha=0.01)$, the number of necks is varying between 60 and 94 , somehow not so far from $n_{\text {dom }}$. Clearly, inertia is structuring the multiple necking pattern, while the initial perturbation just slightly influences the final distribution of necks. These findings illustrate regions I and III of Fig. 5. On the other hand, when $N_{0}=50$ and $\alpha=0.05$, it is observed that the final number of necks is close to the initial pattern.
This situation corresponds to the region II of Fig. 6. To summarize :

- if the initial spectrum is sharp and $N_{0}$ is far from the dominant pattern given by the white noise case $n_{d o m}^{w n}$, the perturbation evolves rapidly towards this pattern and the final number of necks approaches $n_{d o m}^{w n}\left(n_{d o m} \approx n_{d o m}^{w n}\right)$;

- if the initial spectrum is large or if $N_{0}$ is close to $n_{d o m}^{w n}$, the evolution of the pre-neck spacing distribution is slow. The final number of necks stays close to $N_{0}\left(n_{d o m} \approx N_{0}\right)$.

\section{Conclusion}

An extension of the Linear Stability Analysis (LSA) developed in [6] applied to the development of the instabilities in a bar in dynamic tension has been proposed. The history of the development of the instabilities and the multi-modal aspect of the perturbations can now be considered. Besides, a new statistical approach based on the power spectrum of the perturbations is also proposed to evaluate the neck spacing.

From this approach, the effect of the loading velocity can be captured by the model, evolutions in agreement with experimental results of [7] are obtained. Finally, trends observed in [8] from numerical simulations have been retrieved by imposing a new type of initial perturbation which selects an initial domain of dominant perturbation modes.

The major result of this extension of the classical linear stability analysis comes from the fact that the mean pre-neck spacing is still accurately estimated but also the variability around this dominant mode. The dispersion in fragment size observed in experiments can therefore be better anticipated by the model. 
Table 1. Effect of different initial perturbation on the final dominant mode. The results of Figs.7(a)-7(d) are compared to the one obtained with a white noise perturbation, see Fig.2(a), in terms of dominant pre-neck spacing mode at

\begin{tabular}{|c|c|c|c|c|c|}
\hline & $N_{0}$ & $\alpha$ & $n_{d o m}(20 \mu s)$ & $n_{d o m}(50 \mu s)$ & $n_{d o m}(80 \mu s)$ \\
\hline Fig.2(a) & - & - & N.A. & 71 & 80 \\
\hline Fig.7(a) & 5 & 0.05 & 10 & 12 & 75 \\
\hline Fig.7(b) & 50 & 0.05 & 51 & 52 & 54 \\
\hline Fig.7(c) & 5 & 0.01 & 40 & 48 & 60 \\
\hline Fig.7(d) & 250 & 0.01 & 256 & 255 & 94 \\
\hline
\end{tabular}

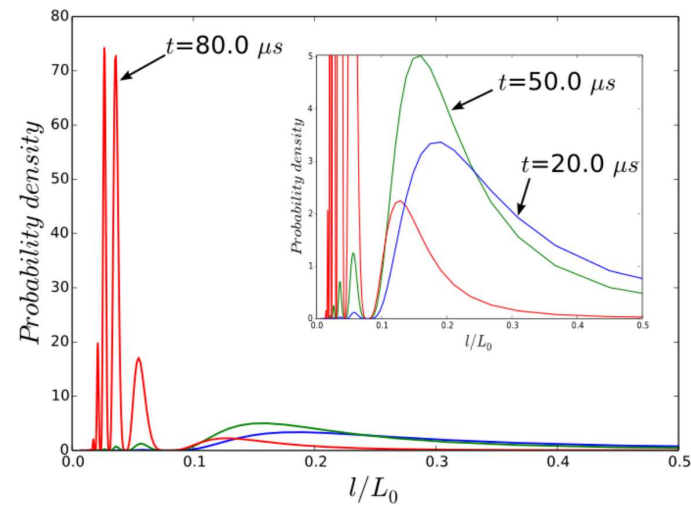

(a) $p_{0}=5, \alpha=0.05$

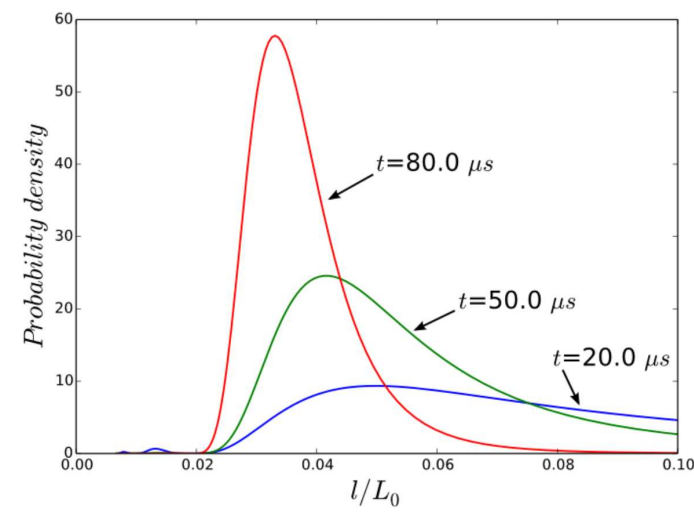

(c) $p_{0}=5, \alpha=0.01$

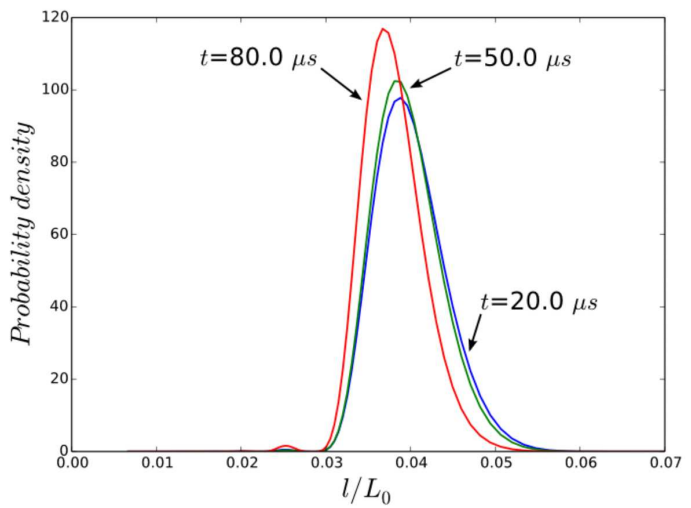

(b) $p_{0}=50, \alpha=0.05$

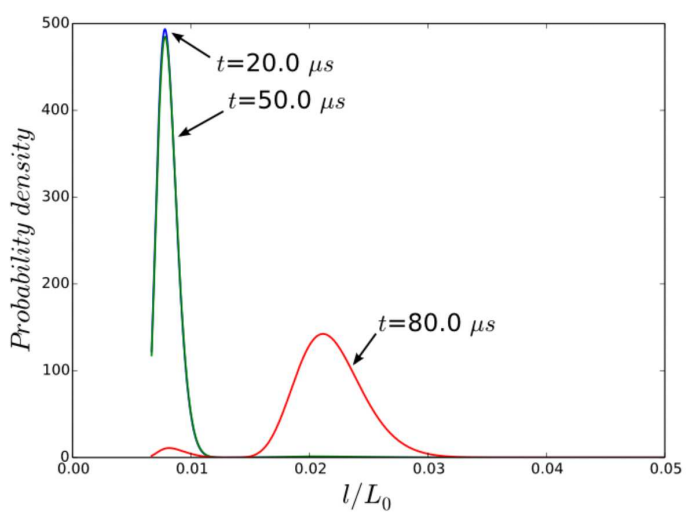

(d) $p_{0}=250, \alpha=0.01$

Fig. 7. Pre-neck spacing probability density of the cross-section fluctuation, for a loading velocity $V_{0}=900 \mathrm{~m} / \mathrm{s}$, at the different times $t=20 \mu \mathrm{s}, t=50 \mu \mathrm{s}$ and $t=80 \mu \mathrm{s}$, for initial perturbations defined by Eq.(5) with the parameters (a) $p_{0}=5, \alpha=0.05$, (b) $p_{0}=50, \alpha=0.05$, (c) $p_{0}=5, \alpha=0.01$ and (d) $p_{0}=250, \alpha=0.015$.

\section{References}

1. N.F. Mott and E.H. Linfoot, Ministry of Supply, AC3348 (1943)

2. G.I. Taylor, Scientific Papers of G.I. Taylor, (Vol. III, No. 44, Cambridge Univ. Press, 1963)

3. D.E. Grady, M.E. Kipp and D.A. Benson, Proc. Conf. Mech. Prop. High Rates of Strain, Oxford, Inst. Phys. Conf. Ser. No. 70, 315-320 (1984)
4. S. El Maï, S. Mercier, J. Petit, A. Molinari, Int. J. Sol. Struct. 51, 3491-3507 (2014)

5. S. El Maï, PhD. Thesis, Univ. de Lorraine, (2014)

6. S. Mercier and A. Molinari, Int. J. Sol. Struc. 40, 1995-2016 (2003)

7. H. Zhang, K. Ravi-Chandar, Int. J. of Frac., 142, 183-217(2006)

8. J. Rodríguez-Martínez, G. Vadillo, R. Zaera, J. Fernández-Sáez, Mech. Mat., 60, 107-120 (2013) 